\title{
1-Deoxy-D-Xylulose 5-Phosphate Reductoisomerase, a Key Target for Novel Antibacterial Development against Acinetobacter baumannii and Klebsiella pneumoniae
}

\author{
H Ball ${ }^{1}$, M Girma ${ }^{2}$, M Zainab ${ }^{2}$, I Soojhawon ${ }^{3}, \mathrm{R} \mathrm{Couch}^{2}$, S Noble ${ }^{3}$ \\ ${ }^{1}$ Walter Reed Army Institute of Research, Centreville, VA, ${ }^{2}$ George Mason University, Manassas, \\ VA, ${ }^{3}$ Walter Reed Army Institute of Research, Silver Spring, MD \\ HALEY.SIAN.BALL@GMAIL.COM
}

Acinetobacter baumannii and Klebsiella pneumoniae are members of the ESKAPE pathogens (Enterococcus faecium, Staphylococcus aureus, K. pneumoniae, A. baumannii, Pseudomonas aeruginosa, and Enterobacter species), a group of multidrug-resistant bacteria which are currently the leading cause of nosocomial infections worldwide. Presently, carbapenem-resistant A. baumannii and K. pneumoniae are classified as urgent threats by the Centers for Disease Control. The bacterial methylerythritol phosphate (MEP) pathway presents an attractive target for antibiotic development, as it is essential for production of vital isoprenoids including ubiquinone, menaquinone, bactoprenols, and bacterial hopanoids. The corresponding mammalian pathway of isoprenoid biosynthesis, the mevalonic acid pathway, is distinct from the MEP pathway. To facilitate antibiotic development against A. baumannii and K. pneumoniae we characterized the first committed MEP pathway enzyme, 1-deoxy-D-xylulose 5phosphate reductoisomerase (IspC), of $\mathrm{A}$. baumannii and $\mathrm{K}$. pneumoniae. We assessed in vitro IspC activity inhibition and performed antimicrobial susceptibility testing with two IspC inhibitors, fosmidomycin and FR900098. A baumannii and K. pneumoniae IspC activity was significantly inhibited by fosmidomycin and FR900098, with IC50 values between 19.5 - $45.5 \mathrm{nM}$. A. baumannii strains AB5075 and AB19606 were susceptible to FR900098 with minimum inhibitory concentrations (MICs) of 256 and $128 \mu \mathrm{g} / \mathrm{ml}$ respectively. The growth of K. pneumoniae strains Kp_NSC-277 and Kp_BAA-1705 was inhibited by both FR900098 and fosmidomycin with MICs in the range of $64-256 \mu \mathrm{g} / \mathrm{ml}$. Previous studies have shown that cellular penetration of fosmidomycin and FR900098 is limited by their polar nature and/or by cellular uptake via the glycerol 3-phosphate transporter, which may account for the relatively high MICs observed in this study. However, increasing the lipophilicity of fosmidomycin analogs and/or employing a phosphate diester prodrug strategy has been shown to significantly improve their antibacterial activity. In order to enhance inhibitor development, we determined the 2.5 $\AA$ crystal structure of IspC from A. baumannii in complex with inhibitor, FR900098, and cofactors NADPH and magnesium. Given that IspC activity was significantly inhibited by fosmidomycin and FR900098, and bacterial growth was inhibited, to a lesser extent, by fosmidomycin or FR900098, we conclude that IspC of A. baumannii and $\mathrm{K}$. pneumoniae is a valid target for antibiotic development. The elucidation of the enzyme-inhibitor binding interactions presented herein will aid structure-based drug design and prodrug strategies for design and development of derivatives with improved antibacterial activity.

Acta Cryst. (2020). A76, a44 